between the two methods over the range 2.6-4.6 $\log _{10}$ [cells $\left./ 100 \mathrm{ml}\right]$.

The authors conclude that the Bioplorer ${ }^{\circledR}$ apparatus can be used to rapidly detect microbial contamination of dialysate.

Original article Shimakita T et al. (2007) Rapid count of microbial cells in dialysate. Ther Apher Dial 11:363-369

\section{Aspirin prescription does not reduce the risk of cardiac events in patients on hemodialysis}

Patients receiving maintenance hemodialysis are at high risk for cardiovascular disease. In the general population, aspirin is effective for both the primary and secondary prevention of cardiovascular disease. Although the efficacy of this agent in patients with chronic renal disease is unknown, a Task Force convened by the National Kidney Foundation concluded that it would be reasonable to prescribe antiplatelet drugs to patients on hemodialysis with, or at high risk for, coronary artery disease on the basis of the benefits seen in the general population. The results of a cohort study published in the American Journal of Kidney Diseases do not support this assumption.

The researchers examined the patterns and benefits of antiplatelet prescription in 28,320 hemodialysis patients randomly selected from the Dialysis Outcomes and Practice Patterns Study. The average prevalence of aspirin prescription varied widely between countries, from $8 \%$ to $40 \%$ of patients. Older age, male sex, coronary artery disease, hypertension, cerebrovascular disease, peripheral vascular disease and diabetes were all associated with an increased likelihood of aspirin prescription. Aspirin prescription reduced the risk for stroke (relative risk [RR] 0.82; $P=0.03$; comparison with no aspirin prescription), but was associated with increased risks for myocardial infarction (RR 1.21; $P<0.01)$ and any cardiac event (RR 1.08; $P<0.01)$. No associations were found between aspirin prescription and the risks of transient ischemic attack, allcause mortality, all-cause hospitalization or gastrointestinal bleeding.

Original article Ethier J et al. (2007) Aspirin prescription and outcomes in hemodialysis patients: the Dialysis Outcomes and Practice Patterns Study (DOPPS). Am J Kidney Dis 50: 602-611

\section{Why do dialysis patients with AMI have such poor outcomes?}

Acute myocardial infarction (AMI) in dialysis patients is associated with high 1-year and 2-year mortality. Despite the introduction of reperfusion therapy, mortality has remained the same since the 1970s. In an attempt to elucidate factors that could be responsible for the poor long-term outcome of this patient population, Herzog et al. designed a collaborative retrospective cohort study with the National Registry of Myocardial Infarction (NRMI) and the Cardiovascular Special Studies Center of the US Renal Data System (USRDS).

Cross matching of AMI-related hospitalizations from the USRDS database with the NRMI over a 2-year period identified 3,049 patients, who formed the study cohort. Dialysis and nondialysis patients with $\mathrm{AMl}$ exhibited significant clinical differences: $44.4 \%$ of dialysis patients presented with chest pain versus $68.3 \%$ of nondialysis patients. Furthermore, $19.1 \%$ of patients undergoing dialysis had ST-segment elevation AMI, versus $35.9 \%$ of nondialysis patients. Unsurprisingly, therefore, $44.8 \%$ of dialysis patients were diagnosed as not having acute coronary syndrome on admission, compared with $21.2 \%$ of nondialysis patients. These misdiagnoses had serious consequences: cardiac arrest and in-hospital death were both twice as frequent in patients requiring dialysis than in those who did not undergo dialysis.

The authors conclude that dialysis patients hospitalized for AMI present with strikingly different signs and symptoms to nondialysis patients, which could explain their poor outcomes. They recommend intensive efforts for early, accurate recognition of $\mathrm{AMI}$ in dialysis patients.

Original article Herzog CA et al. (2007) Clinical

Characteristics of Dialysis Patients With Acute Myocardial Infarction in the United States: a collaborative project of the United States Renal Data System and the National Registry of Myocardial Infarction. Circulation 116: 1465-1472

\section{Hemodialysis patients with low vitamin D levels are at increased risk of early mortality}

Dysfunctional kidneys often lack the capacity to convert 25-hydroxyvitamin $D$ (the storage form of vitamin D) into its biologically active form, 1,25-dihydroxyvitamin $\mathrm{D}$, which commonly 